\title{
CARACTERÍSTICAS PRODUCTIVAS EN POLLOS DE ENGORDE UTILIZANDO HARINA DE ORÉGANO COMO PROMOTOR DE CRECIMIENTO
}

\section{PRODUCTIVE CHARACTERISTICS IN BROILERS USING OREGANO FLOUR AS A GROWTH PROMOTER}

\author{
Juan Tenías Campos ${ }^{1}$, Mayra Alfaro Escalona ${ }^{1}$, Magalys Rivas Nichorzon ${ }^{1}$, Liseth Cárdenas Ramírez ${ }^{1}$ y Ramón Silva- \\ Acuña $^{2}$
}

${ }^{1}$ Universidad de Oriente, Núcleo Monagas, Escuela de Zootecnia, Campus Los Guaritos, Maturín, Monagas, Venezuela

${ }^{2}$ Universidad de Oriente, Núcleo de Monagas, Escuela de Ingeniería Agronómica, Postgrado de Agricultura Tropical, Campus Juanico, Maturín, Venezuela

E-mail: drramonsilvaa@gmail.com

\section{Información del artículo}

Tipo de artículo: Artículo original

Recibido:

28/06/2021

Aceptado:

02/11/2021

Licencia:

CC BY-NC-SA 4.0

Revista ESPAMCIENCIA 12(2):107-115

DOI:

https://doi.org/10.5 1260/revista_espam ciencia.v12i2.283

\section{Resumen}

Con el objetivo de evaluar la inclusión de proporciones de harina de hojas de orégano (HHO) en el alimento balanceado comercial (ABC) de pollos de engorde, se cuantificó en fase de inicio y de engorde, el peso corporal (PC), ganancia diaria de peso (GDP), consumo diario de alimento (CDA), índice conversión alimenticia (ICA) y mortalidad (MO) en un diseño experimental completamente al azar. Se utilizaron 208 pollitos de la línea Ross distribuidos en cuatro tratamientos: $\mathrm{ABC} 100 \%$ : $100 \%$ de alimento balanceado; $\mathrm{ABC}+0,25 \mathrm{HHO}$ : Alimento balanceado $+0,25 \%$ de harina de orégano; $\mathrm{ABC}+0,50 \mathrm{HHO}$ : Alimento balanceado $+0,50 \%$ de harina de orégano y $\mathrm{ABC}+0,75 \mathrm{HHO}$ : Alimento balanceado $+0,75 \%$ de harina de orégano, con cuatro repeticiones y 13 aves por unidad experimental. Las variables PC y GDP fueron analizadas por regresión, mientras que CDA, ICA y MO fueron comparadas por estadística descriptiva. El PC logró diferencias significativas al final de las fases de inicio y engorde, siendo el tratamiento $\mathrm{ABC}+0,25 \mathrm{HHO}$, obteniendo el mejor resultado $(773,18$ y $2196,11 \mathrm{~g}$, respectivamente). La GDP siguió la misma tendencia del $\mathrm{PC}$, donde el tratamiento $\mathrm{ABC}+0,25 \mathrm{HHO}$ mostró el mayor valor, con 35,01 y 67,71g a los 21 y 42 días, respectivamente. Las variables CDA e ICA se vieron favorecidas con la dieta $\mathrm{ABC}+0,25 \mathrm{HHO}$. No se encontraron diferencias estadísticas para la mortalidad. La adición de $0,25 \%$ de harina de hojas de orégano (HHO), como aditivo fitogénico en la dieta de pollos de engorde, tuvo efecto positivo sobre el peso corporal, ganancia diaria de peso, consumo de alimento y conversión alimenticia durante la fase de inicio y engorde.

Palabras clave: Origanum vulgare, aditivo de crecimiento, fitogénico, aves de corral.

\section{Abstract}

In order to evaluate the inclusion of proportions of oregano leaf flour (HHO) in the commercial balanced feed $(\mathrm{ABC})$ of broilers, the body weight $(\mathrm{CP})$, daily gain were quantified in the start and fattening phase weight (GDP), daily feed intake (ADC), feed conversion index (ICA) and mortality (OM) in a completely randomized experimental design. 208 chicks of the Ross line were used distributed in four treatments: $\mathrm{ABC} 100 \%: 100 \%$ balanced feed; $\mathrm{ABC}+0.25 \mathrm{HHO}$ : Balanced feed $+0.25 \%$ oregano flour; $\mathrm{ABC}+0.50 \mathrm{HHO}$ : Balanced feed + $0.50 \%$ oregano flour and $\mathrm{ABC}+0.75 \mathrm{HHO}$ : Balanced feed $+0.75 \%$ oregano flour, with four repetitions and 13 birds per experimental unit. The variables PC and GDP were analyzed by regression, while CDA, ICA and MO were compared by descriptive statistics. The $\mathrm{CP}$ achieved significant differences at the end of the start-up and fattening phases, being the treatment $\mathrm{ABC}+0.25 \mathrm{HHO}$, the one that obtained the best result $(773.18$ and $2196.11 \mathrm{~g}$, respectively). The GDP followed the same trend as the PC, where the treatment $\mathrm{ABC}+0.25 \mathrm{HHO}$ showed the highest value, with 35.01 and $67.71 \mathrm{~g}$ at 21 and 42 days, respectively. The variables CDA and ICA were favored with the $\mathrm{ABC}+0.25 \mathrm{HHO}$ diet. No statistical differences were found for mortality. The addition of $0.25 \%$ oregano leaf flour (HHO), as a phytogenic additive in the diet of broilers, had a positive effect on body weight, daily weight gain, feed consumption and feed conversion during the starting and fattening phase.

Keywords: Origanum vulgare, growth additive, phytogenic, poultry. 


\section{INTRODUCCIÓN}

La producción mundial de carne de pollo representa el $30 \%$. Estados Unidos es el principal productor, con el $26 \%$ del total; le continúan, China 17\%, Brasil 16\%, Unión Europea con $14 \%$, México 4\%, India 3\%, mientras que Japón, Argentina y Canadá poseen porcentajes similares al 2\% (Lara et al., 2009).

El aspecto de mayor importancia en la avicultura es la alimentación. Las aves deben recibir el alimento en cantidad y calidad suficiente que contenga proporciones adecuadas de los nutrimentos necesarios para que obtengan el rendimiento apropiado en carne (Roldán, 2004). En el pasado esos alimentos poseían promotores de crecimiento (APC); la OMS (2017) los define como "sustancias distintas de los nutrimentos de la ración que aumentan el ritmo de crecimiento y mejoran el índice de conversión de los animales sanos y correctamente alimentados". El término promotor del crecimiento se puede aplicar a más de un tipo de sustancia usada en producción animal.

Ávila (1990), describe a los antibióticos como mejoradores de crecimiento, estos productos cuando son aplicados o suministrados a los animales, tienen la capacidad de prevenir el establecimiento de microorganismos patógenos dentro del microbiota intestinal; además, de proporcionar ganancias marcadas en los incrementos de masa, desarrollo y crecimiento muscular. Los promotores de crecimiento como aditivos en el alimento en concentraciones subterapéuticas, aumentan el rendimiento y la productividad de los animales a través del control de bacterias patógenas, inhibiendo su crecimiento o controlándolas, manteniendo sano el tracto digestivo del animal y en consecuencia, mejor aprovechamiento de los nutrimentos contenidos en los alimentos (Lee et al., 2003). Por otro lado, Ayala et al. (2006), indican que la creciente preocupación de los consumidores sobre el posible traslado de la resistencia antibiótica a los patógenos causantes de enfermedades humanas, ha provocado la prohibición de la mayoría de los antibióticos promotores de crecimiento.

Ortisi (2008), señala que los promotores de crecimiento utilizados en bajas dosificaciones, mejoran la calidad del producto final, traducida en menor proporción de grasa y mayor proporción de proteínas. Otro beneficio de la utilización de estos fármacos en la dieta es el control de patógenos zoonóticos, tales como Salmonella sp. , Campylobacter sp., Escherichia coli y Enterococos, entre otros. Con menos bacterias en el tracto, hay menor producción de toxinas bacterianas; amoníaco, nitratos, aminas, y otros, que producen las bacterias consideradas tóxicas para las células intestinales, también pueden absorberse a la sangre y causar problemas en otras partes del cuerpo (Quintana, 1993).

\section{ERSPAMACIENCIA}

La industria avícola dejó de usar los antibióticos como promotores de crecimiento para sustituirlos por lo probióticos, enzimas, acidificantes -ácidos orgánicos e inorgánicos-y extractos vegetales (González et al., 1999; Carro y Ranilla, 2002).

Los extractos vegetales o compuestos fitogénicos poseen ingredientes funcionales con acciones antimicrobianas, antioxidantes e inmunomoduladoras (Dewick, 1997; Garcés et al., 2009; Díaz, 2015). Los metabolitos secundarios, podrían ser de diversos tipos: alcaloides, polifenoles y terpenoides, responsables de estos efectos beneficiosos (Quintana, 1993). Kokkini et al. (1997), definen los fitogénicos como sustancias naturales provenientes de las plantas, que usadas en cantidades terapéuticas ocasionan resultados favorables para los animales, aumentando su capacidad productiva.

Autores como Devriese et al. (1993) y Lee et al. (2003) reportan el efecto beneficioso con el uso de las combinaciones de harinas de hojas de plantas aromáticas similar a los encontrados en promotores de crecimiento, en razón del eficiente uso de los nutrimentos de la dieta, que se traduce en menor conversión de alimento. Agrega Kamel (2001), que el desarrollo de vellosidades intestinales y el estímulo de la actividad enzimática están directamente involucrados y afectan el consumo de alimento.

Garcés et al. (2009), indican diferentes sustancias con actividad antibacteriana, antiparasitaria y antimicótica de algunas plantas, entre ellas el orégano. En cuanto a la composición del orégano, se ha logrado identificar gran cantidad de compuestos y sólo se han encontrado como componentes principales activos a las sustancias fenólicas que alteran la permeabilidad de la membrana celular de bacterias patógenas como las Salmonelas y Escherichia coli, responsables de trastornos digestivos en las categorías menores porcinas. De ahí que, la obtención a gran escala, y el empleo en la alimentación resulta de gran interés para la producción animal (Barragán et al., 2004).

El uso del orégano en la producción animal es diverso, una de ellas lo constituye la extracción de su aceite esencial, esta forma se ha incrementado considerablemente en los últimos tiempos demostrando la actividad biológica de sus componentes con muy buenos resultados. Recientemente, Campozano-Marcillo et al. (2020), evaluaron la inclusión de aceites esenciales de orégano en concentraciones de $100 ; 200$ y $300 \mathrm{mg} \cdot \mathrm{Kg}^{-1}$ del alimento balanceado, constatando que la mayor concentración favoreció el peso vivo, la ganancia de peso y la conversión alimenticia de los pollos de engorde.

Por otro lado, Botsoglou et al. (2002), recalcan que no solo el aceite constituye una forma eficiente de utilización, las hojas frescas incluidas en la dieta han sido de gran aprobación, debido a que mejora la aceptabilidad de los alimentos con resultados beneficiosos de manera general 
en el comportamiento productivo e indicadores de salud de los animales monogástricos. Otra forma de aplicación ha sido la obtención de harina de orégano a partir de hojas secadas y molidas con niveles bajos de inclusión hasta de $1 \%$ en la dieta.

Carro y Ranilla (2002), determinaron el comportamiento productivo de pollos de engorde, incluyendo $0 ; 0,5$ y $1 \%$ de orégano en su dieta. Los resultados mostraron que no hubo diferencias para el peso vivo $(1714,1690$ y $1680 \mathrm{~g})$ pero con la inclusión de $0,5 \%$ de orégano, el consumo de alimento fue menor que al incluir 1\% (3706; 3461 y 3578 g.ave $\left.{ }^{-1}\right)$, mientras que el grupo control presentó mayor conversión $(2,15 ; 2,08$ y 2,10), mayor consumo de proteína $(648,601$ y $627 \mathrm{~g})$ y mayor conversión proteica $\left(0,387 ; 0,361\right.$ y $0,379 \mathrm{~g} \cdot \mathrm{g}^{-1}$ para $0 ; 0,5$ y $1 \%$ de orégano en la dieta, respectivamente). Estos resultados sugieren que es factible incluir en la dieta aditivos fotogénicos como el orégano.

Ayala et al. (2006), evaluaron el comportamiento productivo y el rendimiento de la canal en pollos de engorde, con el uso de harina de hojas de plantas aromáticas como aditivo fitoterapéutico al $0,07 \%$ en la dieta. A los 21 días de edad, los pollos alimentados con Origanum vulgare tuvieron el menor peso corporal y alta conversión alimenticia de los tratamientos durante esta fase $(p \leq 0,05)$. El testigo (flavomicina $4 \%$ ) registró la mayor mortalidad a los mismos días de edad. Al final de la prueba (42 días), el mayor peso corporal lo obtuvo el mismo testigo (flavomicina $4 \%$ ), pero no presentó diferencia $(p \leq 0,05)$ frente los alimentados con Origanum vulgare y tuvo la menor conversión de alimento. El uso de estas harinas permite obtener idénticos resultados a los alcanzados con los promotores de crecimiento comerciales; de manera similar, al evaluar proporciones de mezcla del alimento balanceado y orégano seco y molido al 0,$5 ; 1,0$ y $1,5 \%$, Pujada et al. (2019) constataron que el nivel óptimo de orégano en la dieta fue de $0,71 \%$.

Tomando como referente los anteriores hallazgos, en este artículo se presenta el resultado de la evaluación del comportamiento productivo de pollos de engorde de la línea Ross, utilizando harina de hojas de orégano (Origanum vulgare) en proporciones de mezcla con el alimento balanceado, como promotor de crecimiento.

\section{MATERIALES Y MÉTODOS}

\section{Ubicación del ensayo}

El ensayo se realizó en una granja comercial ubicada en la localidad de Tácata, municipio Cedeño, estado Monagas. En las coordenadas geográficas $9^{\circ} 63^{\prime} 77^{\prime \prime} \mathrm{LN}$ y $63^{\circ} 67^{\prime}$ 13 " LO, a la altitud de $124 \mathrm{~m}$, con temperatura promedio de $27^{\circ} \mathrm{C}$, humedad relativa de $63 \%$ y precipitación promedio anual de $1298 \mathrm{~mm}$ (Climate-Data.org, 2020).

\section{El orégano y su preparación}

El material vegetal de orégano, se obtuvo de un Jardín Botánico ubicado en la localidad de Temblador, municipio Libertador, estado Monagas, en las coordenadas geográficas de $9^{\circ} 00^{\prime} 26^{\prime \prime} \mathrm{LN}$ y $62^{\circ} 38^{\prime} 32^{\prime \prime}$ LO. El follaje se cortó con tijeras de podar y tijeras comunes; se secó al sol sobre un plástico negro por 72 horas y posteriormente fue molido en un triturador de granos marca GENPAR, modelo GGG-121-HH, Venezuela, en la regulación fina, con el objetivo de lograr su mezcla homogénea con el alimento balanceado. El orégano molido se almacenó en envases de vidrio sellados.

Entre las especies de Origanum se encuentran como componentes principales el limoneno, el b-cariofileno, el r-cimeno, el canfor, el linalol, el a-pineno, el carvacrol y el timol (Arcila-Lozano et al., 2004).

\section{Acondicionamiento del galpón para la recepción}

Previo a la llegada de los pollitos bebé, para iniciar el ensayo se realizó la limpieza y desinfección del galpón experimental y todos los equipos. Se colocaron cortinas de polietileno alrededor del lugar, para proteger a las aves del frío y viento. Posteriormente se realizaron las respectivas divisiones e identificaciones para los tratamientos, utilizando pisos plásticos para bovinos, de $100 \mathrm{~cm}$ de largo por $60 \mathrm{~cm}$ de ancho.

Finalmente, se colocó la cama de cascarilla de arroz y se instalaron bebederos que contenían agua más hidratante comercial para disminuir el estrés en la recepción; además, del alimento iniciador en comederos manuales. Se utilizó una criadora a gas Marca Gasolec M8, modelo GA801351, como fuente de calor durante los primeros días de vida de los pollitos. El consumo de agua fue ad libitum durante todo el ensayo, utilizando bebederos manuales.

\section{Manejo sanitario de las aves}

A las aves se las recibió vacunadas contra la enfermedad de Marek, desde la planta incubadora. El manejo sanitario preventivo consistió en una serie de medidas aplicadas a diferentes días de edad, a partir de su recepción, que consideraron la aplicación de las vacunas obligatorias de la zona. Entre los 1 y 3 días, recibieron electrolitos en la dosis de $1 \mathrm{~L} .1000 \mathrm{~mL}^{-1}$ de agua; al octavo y 16 día, se les aplicó la primera y segunda dosis, respectivamente, contra New Castle (Cepa La Sota) y Gumboro, mediante aspersión. A los 10 días y entre los 22 y 24 días de edad, se les ofreció tilosina en dosis de $250 \mathrm{~g} .1000 \mathrm{~mL}^{-1}$ de agua. Entre los 11 y 13 días, recibieron polivitamínico en la dosis de $1 \mathrm{~L} .1000 \mathrm{~mL}^{-1}$ de agua y vinagre a razón de 1L. $1000 \mathrm{~mL}^{-1}$ a los 21 días de edad.

\section{Variables cuantificadas}

El peso corporal (PC), se determinó al término de la fase de inicio (21 días) y engorde (42 días). Todos los pesajes se hicieron a la misma hora ( $2 \mathrm{pm})$ tomando como muestra 
seis de la totalidad de los pollitos presentes en cada unidad experimental. El peso corporal promedio se obtuvo utilizando la ecuación 1.

$$
\mathbf{P C}=\sum \text { Peso de cada ave } / \mathrm{N}^{\circ} \text { de aves (1) }
$$

La ganancia diaria de peso (GDP), se obtuvo por diferencia entre el peso inicial y el final de la fase de evaluación, dividido por la duración del periodo comprendido. Este valor se registró durante la fase de inicio (21 días) y engorde (42 días), utilizando la ecuación 2.

$$
\mathbf{G D P}=(\text { peso promedio final }- \text { peso promedio inicial }) / \mathrm{N}^{\circ} \text { de días (2) }
$$

El porcentaje de mortalidad se determinó por la ecuación 3 .

MO $(\%)=\left(\mathrm{N}^{\circ}\right.$ de aves muertas $/ \mathrm{N}^{\circ}$ de aves iniciadas $) \times 100(3)$

El consumo diario de alimento (CDA), representa la cantidad del alimento consumido por las aves entre el total de las aves vivas, calculado por la ecuación 4 .

CDA= Alimento consumido total $(\mathrm{g}) / \mathrm{N}^{\circ}$ de aves (4)

El índice de conversión alimenticia (ICA), expresa la eficiencia del alimento para su transformación en carne, por consiguiente, cuanto más bajo sea este índice, resulta mejor. Oscila entre 1,6 a 1,7 kg de alimento consumido.kg${ }^{1}$ de peso producido (Barragán et al., 2004) y determinado por la ecuación 5 .

ICA= Alimento consumido por ave (g) / peso del ave (g) (5)

\section{Tratamientos evaluados, diseño experimental y análisis estadístico}

Los componentes del alimento balanceado comercial Mersan $^{\circledR}(\mathrm{ABC})$ son los siguientes: fórmula de inicio, energía metabolizable mínimo $3100 \mathrm{Kcal} \cdot \mathrm{Kg}^{-1}$; proteína mínimo $22 \%$; grasa mínimo $2,5 \%$; fibra máximo $5 \%$; calcio mínimo $0,95 \%$ y fósforo disponible mínimo 0,35 $\%$, y la de engorde, energía metabolizable mínimo 3300 Kcal. $\mathrm{Kg}^{-1}$; proteína mínimo $19 \%$; grasa mínimo $6,5 \%$; fibra máximo $4,5 \%$; calcio mínimo $0,85 \%$ y fósforo disponible mínimo $0,32 \%$; y la harina de orégano (HHO). Los tratamientos fueron los siguientes: $\mathrm{ABC} 100 \%: 100 \%$ de alimento balanceado; $\mathrm{ABC}+0,25 \mathrm{HHO}$ : Alimento balanceado $+0,25 \%$ de harina de orégano; $\mathrm{ABC}+0,50 \mathrm{HHO}$ : $50 \%$ de alimento balanceado $+0,50 \%$ de harina de orégano; y $\mathrm{ABC}+0,75 \mathrm{HHO}: 25 \%$ de alimento balanceado $+0,75 \%$ de harina de orégano.

Por kilogramo de la mezcla $\mathrm{ABC}+\mathrm{HHO}$, tanto para el alimento iniciador como de engorde, las proporciones de mezclas corresponden a: $997,5+2,5 \mathrm{~g}$; $995+5 \mathrm{~g}$ y $992,5+7,5$ g para los tratamientos 2,3 y 4 , respectivamente. Se empleó una balanza digital de precisión Gramera, marca Tanita, para el pesaje de la cantidad de orégano en las diferentes dietas alimenticias y un peso digital portátil de gancho $(50 \mathrm{~kg})$, marca Weiheng, para registrar los diferentes pesos de los pollos. El proceso de mezcla del $\mathrm{ABC}$ y la $\mathrm{HHO}$ se efectuó en un galpón completamente limpio, utilizando una cortina de polietileno desinfectada como base, para luego remover de forma manual los dos componentes, hasta lograr la homogenización total.

Se emplearon 208 pollos de la línea Ross, de un día de edad; distribuidos en cuatro tratamientos de 52, en el diseño completamente al azar, con cuatro repeticiones de 13, que constituyen la unidad experimental. Para realizar la investigación se utilizó $24 \mathrm{~m}^{2}$ del área de un galpón de $2250 \mathrm{~m}^{2}$, siendo la densidad de 13 pollos por $\mathrm{m}^{2}$.

Previo a realizar el análisis de varianza (ANAVA) de las variables cuantificadas a los 21 días de la fase de inicio y a los 42 días de engorde, referidas a peso corporal, ganancia diaria de peso y mortalidad se exploraron por la prueba de Shapiro-Wilk para corroborar la normalidad de los errores y la prueba de Bartlett para la homogeneidad de varianza. Los resultados de los análisis estadísticos de las variables peso corporal y ganancia diaria de peso fueron interpretados por regresión. Las variables consumo de alimento y conversión alimenticia se analizaron por estadística descriptiva, en virtud de que el valor obtenido proviene de cuantificaciones hechas por volumen total de alimento consumido ( $\mathrm{kg}$ totales/tratamiento), rutinarias en sistemas industriales de producción. Para ambos análisis se empleó el programa estadístico InfoStat Versión 2018 (Di Rienzo et al., 2011).

\section{RESULTADOS Y DISCUSIÓN}

\section{Peso corporal}

Los resultados obtenidos para peso corporal a los 21 días de edad muestran diferencias significativas entre los tratamientos $(p \leq 0,05)$. En la Figura 1 , se aprecia la tendencia cuadrática obtenida en el análisis de regresión, la cual describe una curva convexa entre los niveles de inclusión de orégano evaluados.

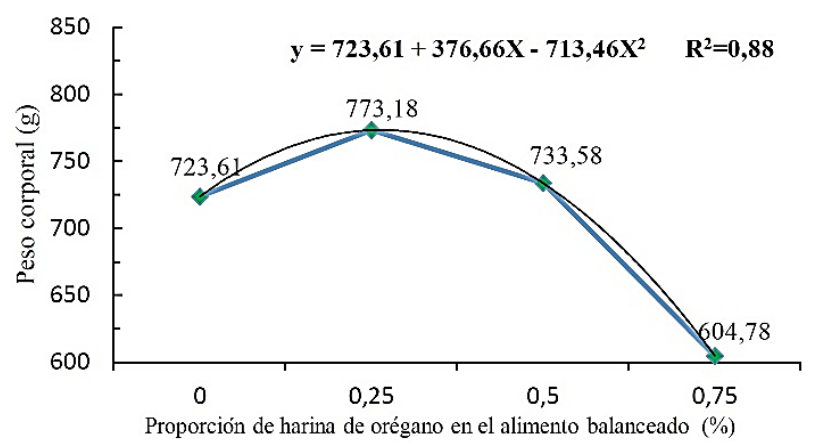

Figura 1. Ecuación de regresión para la variable peso corporal a los 21 días - iniciación-, en función de los niveles de incorporación de harina de orégano. 
Entre 0 y $0,25 \%$ de inclusión, el peso corporal aumentó de 723,61 a $773,18 \mathrm{~g}$, y a partir de $0,25 \%$ se observa disminución progresiva de la variable, pasando con $0,50 \%$ a 733,58g, hasta llegar con $0,75 \%$ de inclusión a $604,78 \mathrm{~g}$. El cálculo de punto máximo de la ecuación, estima que al incorporar $0,26 \%$ de orégano en la dieta, se lograría el mayor peso corporal del ave, de aproximadamente $733,31 \mathrm{~g}$.

Los pesos obtenidos a los 42 días de edad, siguieron la misma tendencia que a los 21 días, donde el tratamiento $\mathrm{ABC}+0,25 \mathrm{HHO}$; presentó el mayor peso promedio $(2196,11 \mathrm{~g})$, con diferencias altamente significativas. De manera similar, la Figura 2 describe una curva convexa entre los niveles de inclusión de orégano evaluados. Entre 0 y $0,25 \%$ de inclusión, el peso corporal aumentó en magnitud de 1980,56 a 2196,11 g, y a partir de $0,25 \%$ se observa disminución progresiva de la variable, pasando con $0,50 \%$ a $2076,44 \mathrm{~g}$, hasta llegar con $0,75 \%$ de inclusión a $1621,56 \mathrm{~g}$. El cálculo de punto máximo de la ecuación, estima que al incorporar $0,26 \%$ de orégano en la dieta, se lograría el mayor peso corporal del ave, de aproximadamente $2199,49 \mathrm{~g}$.

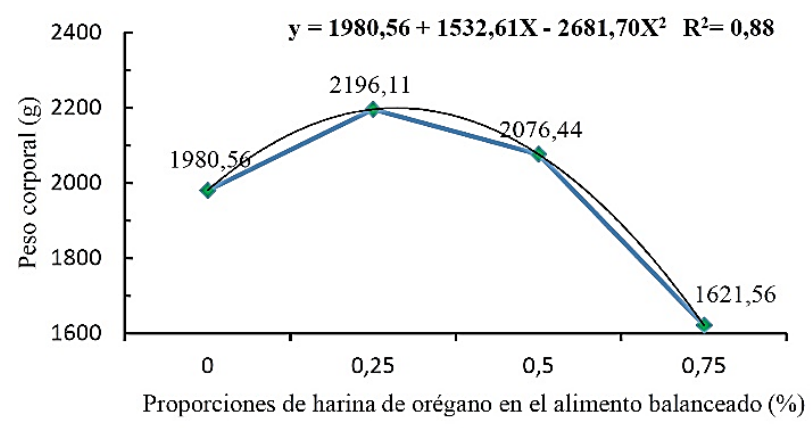

Figura 2. Ecuación de regresión para la variable peso corporal a los 42 días -engorde-, en función de los niveles de incorporación de harina de orégano.

Estos resultados son similares a los reportados con los aceites esenciales de orégano, pues comparten una estructura llamada isopropeno, unidad química de los terpenoides, que derivan en dos fenoles, con propiedades antimicrobianas: Timol y Carvacrol (Ringuelet y Viña, 2013). De acuerdo a la concentración de cada uno de ellos, pueden llegar a disociarse las membranas externas de bacterias Gram negativo como E. coli y Salmonella typhimurium, logrando así mejor absorción de nutrimentos por parte del intestino, repercutiendo positivamente en mayor peso corporal del ave.

Estudios realizados por Lara et al. (2010), evaluando el comportamiento productivo en pollos de engorde, con el uso de harina de hojas de plantas aromáticas como aditivo fitoterapéutico al $0,07 \%$ en la dieta, encontraron que el grupo control obtuvo el mayor peso corporal (2385 g) a los 42 días. De igual manera, a los 21 días los pollos alimentados con $0,35 \mathrm{~g}$ de orégano (Origanum vulgare) y
0,35 g de hierba santa (Piper auritum) tuvieron el menor peso corporal $(395,75 \mathrm{~g})$; sin embargo, concluyeron que el porcentaje de inclusión de harinas fue muy baja y por ende no mostró los resultados esperados.

Ayala et al. (2006), utilizando orégano al 0,5 y 1\%, no encontraron diferencias significativas para peso corporal a los 42 días de edad, con 1690 y 1680 g respectivamente. Lo que demuestra que con una inclusión intermedia de $0,25 \%$ de orégano se pueden lograr resultados satisfactorios.

\section{Ganancia diaria de peso}

La ganancia diaria de peso (GDP) tuvo diferencias altamente significativas a los 21 días de edad. En la Figura 3 , se aprecia la tendencia cuadrática obtenida en el análisis de regresión, la cual describe una curva convexa entre los niveles de inclusión de orégano evaluados. Entre $0 \mathrm{y}$ $0,25 \%$ de inclusión, la GDP aumentó en magnitud de 32,59 a $35,01 \mathrm{~g}$, y a partir de $0,25 \%$ se observa disminución progresiva de la variable, pasando con $0,50 \%$ a $33,11 \mathrm{~g}$, hasta llegar con $0,75 \%$ de inclusión a $26,88 \mathrm{~g}$. El cálculo de punto máximo de la ecuación, estima que al incorporar $0,26 \%$ de orégano en la dieta, se lograría la mayor GDP del ave, de aproximadamente 35,01g.

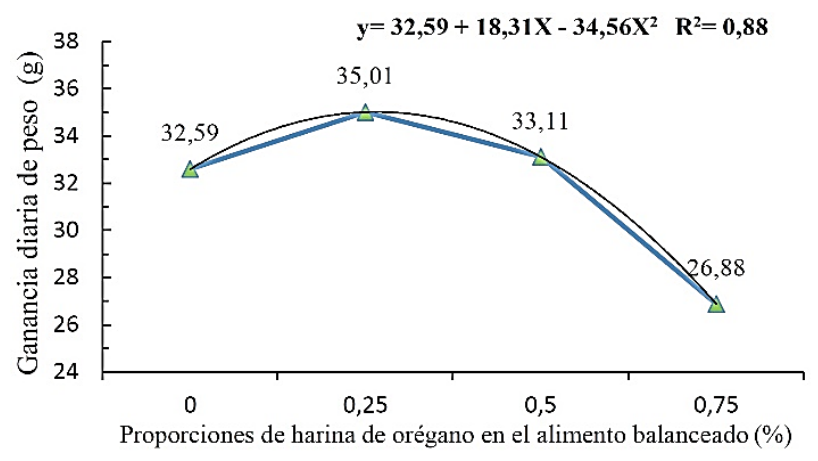

Figura 3. Ecuación de regresión para la variable ganancia diaria de peso a los 21 días -iniciación-, en función de los niveles de incorporación de harina de orégano.

De igual manera, en la Figura 4, se aprecia que al término de la fase de engorde (42 días), existe mayor GDP en el en el tratamiento $\mathrm{ABC}+0,25 \mathrm{HHO}(67,71 \mathrm{~g})$, con diferencias altamente significativas. Seguidos de los tratamientos $\mathrm{ABC}+0,50 \mathrm{HHO} \quad \mathrm{y} \quad \mathrm{ABC} 100 \%$; comportándose estadísticamente similar $(63,91$ y $59,84 \mathrm{~g}$, respectivamente); mientras que el tratamiento $\mathrm{ABC}+0,75 \mathrm{HHO}$ obtuvo las menores ganancias con 48,43 $\mathrm{g}$ al día. El punto máximo de inclusión de orégano, en este caso es de $0,29 \%$, con ganancias máximas de $67,89 \mathrm{~g}$. 


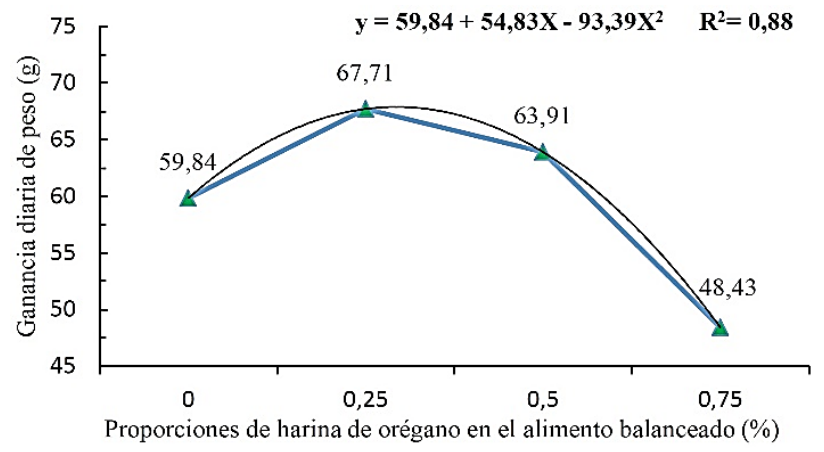

Figura 4. Ecuación de regresión para la variable ganancia diaria de peso a los 42 días -engorde- en función de los niveles de incorporación de harina de orégano.

Estos altos valores en el tratamiento $\mathrm{ABC}+0,25 \mathrm{HHO}$ ) se dieron seguramente por el efecto beneficioso que tienen los componentes del orégano sobre el principal órgano de absorción de nutrimentos, el intestino delgado. El tratamiento $\mathrm{ABC}+0,75 \mathrm{HHO}$, a pesar de contener mayor cantidad de orégano, logró bajos resultados, debido al poco consumo de alimento por parte de las aves.

Hernández et al. (2004) obtuvieron diferencias entre las ganancias diarias de pesos favorables a los extractos de salvia (Salvia officinalis) y de orégano (Origanum vulgare), con respecto al control a los 21 días de edad; sin embargo, a los 42 días no se encontraron diferencias, pero si fueron ganancias mayores ( $83 \mathrm{~g}$ promedio) al presente estudio. Esto parece indicar que los componentes activos de estos aceites que se extrajeron de las plantas ejercen mayor efecto como promotores del crecimiento en pollos de engorde. En el estudio realizado por Chang et al. (2017), utilizando orégano en polvo sobre el rendimiento de crecimiento en pollos de engorde, no se encontraron diferencias significativas para la variable ganancia diaria de peso a los 21 días de edad. No obstante, a los 42 días las aves alimentadas con $150 \mathrm{mg} \cdot \mathrm{kg}^{-1}$ de orégano en la dieta, obtuvieron mayor ganancia $\left(59,47\right.$ g.día $\left.^{-1}\right)$ cuando comparados con el control $\left(54,85\right.$ g.día $\left.{ }^{-1}\right)$.

\section{Consumo diario de alimento}

En la figura 5, se aprecia numéricamente un menor consumo diario de alimento (CDA) en el tratamiento $\mathrm{ABC}+0,75 \mathrm{HHO}(44,70 \mathrm{~g})$, seguidos del tratamiento $\mathrm{ABC}+0,25 \mathrm{HHO}(49,65 \mathrm{~g}), \mathrm{ABC}+0,50 \mathrm{HHO}(49,76 \mathrm{~g})$ y $\mathrm{ABC} 100 \%(50,51 \mathrm{~g})$ a los 21 de edad. De igual manera, en la fase de engorde (22-42 días) el menor consumo fue para el tratamiento $\mathrm{ABC}+0,75 \mathrm{HHO}$ con $103,05 \mathrm{~g}$, comparado con los tratamientos $\mathrm{ABC}+0,25 \mathrm{HHO}, \mathrm{ABC} 100 \%$ y $\mathrm{ABC}+0,50 \mathrm{HHO}$, que obtuvieron un consumo diario de alimento (C) de 124,36, 125,09 y 126,83 g, respectivamente.

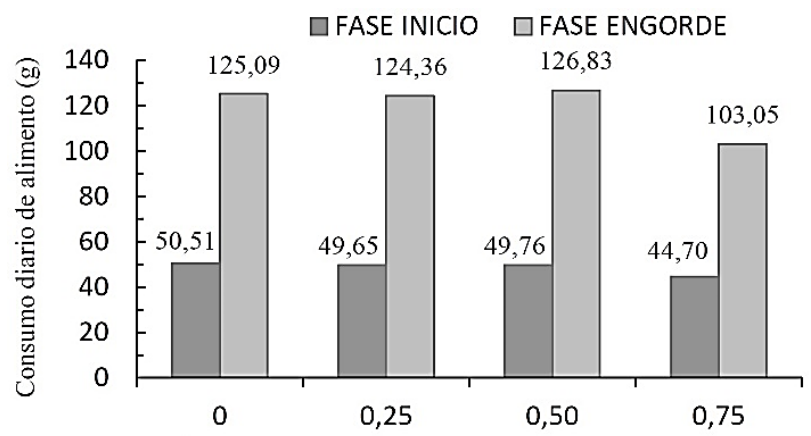

Proporción de harina de orégano en el alimento balanceado (\%)

Figura 5. Histograma del consumo diario de alimento al término de la fase de inicio -21 días - y engorde -42 días-.

Ayala et al. (2006), encontraron diferencias significativas $(p \leq 0,05)$ para el consumo diario de alimento a los 42 días, entre el tratamiento control y el de los pollos que recibieron orégano. El menor consumo se obtuvo con la inclusión de $0,5 \%$ de orégano, mientras que el más alto correspondió al control. Esto quizás se deba por la presencia de metabolitos secundarios como los flavonoides, derivados fenólicos del tipo de los terpenos y sesquiterpenos presentes en el orégano. También podría atribuirse esta restricción de consumo, al cambio sensorial que genera el orégano en la dieta (Windisch et al., 2008; Ordoñez et al., 2018).

Pinchasov y Jensen (1989), Mchan y Shotts (1992) y Eidelsburger y Kirchgessner (1994) plantearon que la inclusión de niveles inferiores a $1 \%$ de sustancias promotoras de crecimiento en aves, apenas tienen efecto en el consumo, mientras que niveles superiores e iguales de $1 \%$ si reducen la ingestión de alimento, tal como ocurrió en el presente estudio.

\section{Índice de conversión alimenticia}

En la Figura 6, se aprecia que el índice de conversión alimenticia (ICA) fue mejor numéricamente para el tratamiento $\mathrm{ABC}+0,25 \mathrm{HHO}$ a los $21(1,31 \mathrm{Ca})$ y $42(1,53$ Ca) días de edad, comparado con los tratamientos $\mathrm{ABC} 100 \%, \mathrm{ABC}+0,50 \mathrm{HHO}$ y $\mathrm{ABC}+0,75 \mathrm{HHO}$, que se comportaron similares al término de las dos fases. Estos valores bajos de conversión alimenticia obtenidos con la inclusión de $0,25 \%$ de orégano en la dieta, se relacionan a que, con este nivel de incorporación, se logró el peso más elevado en cada una de las fases. 


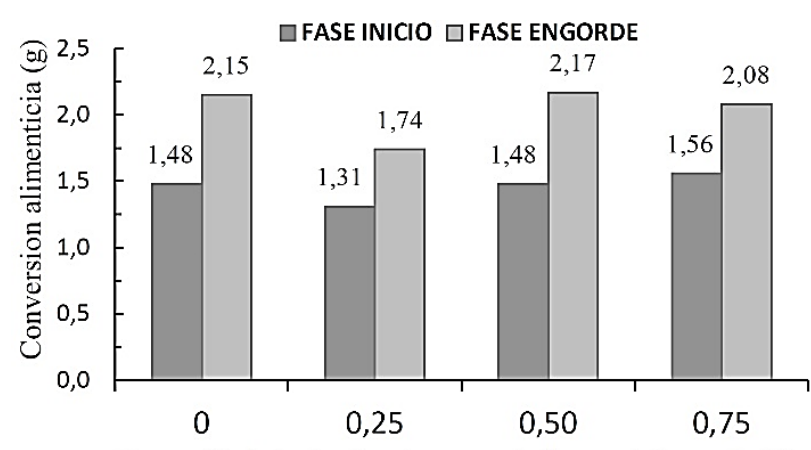

Proporción de harina de orégano en el alimento balanceado (\%)

Figura 6. Histograma para la conversión alimenticia al término de la fase de inicio -21 días- y engorde -42 días-

Karimi et al. (2010), no encontraron diferencias significativas para conversión alimenticia a los 18 días de edad en pollos de engorde que contenían en la dieta diferentes cantidades $\left(2,5 ; 5 ; 10\right.$ y 20 g. $^{-1}{ }^{-1}$ alimento $)$ de orégano contra el control. Los datos que obtuvieron oscilan entre 1,37 y 1,4 ; siendo la dieta con 5 g. $\mathrm{kg}^{-1}$ alimento $(0,5 \%)$ de orégano el menor valor numéricamente. Puvaca et al. (2014), tampoco encontraron diferencias significativas al comparar distintas hierbas de especias (Allium sativum L, Piper nigrum L y Capsicum annuum L) con el control sobre la conversión alimenticia en pollos de engorde a los 42 días de dad, que varío entre 1,8 y 2,1 .

\section{Mortalidad}

No se encontró diferencias estadísticamente significativas para la variable mortalidad en ninguno de los tratamientos; no obstante, en la Figura 7, se observa numéricamente la mayor mortalidad estuvo asociada en los tratamientos $\mathrm{ABC} 100 \%$ y $\mathrm{ABC}+0,50 \mathrm{HHO}$ con $5,77 \%$, seguido de los tratamientos $\mathrm{ABC}+0,25 \mathrm{HHO}$ con $3,85 \%$ y la menor mortalidad ocurrió en el tratamiento $\mathrm{ABC}+0,75 \mathrm{HHO}$ con $1,92 \%$. La mayoría los valores encontrados se ubican por encima del indicador utilizado comúnmente para evaluar la mortalidad en pollos de engorde el cual se establece en $3 \%$.

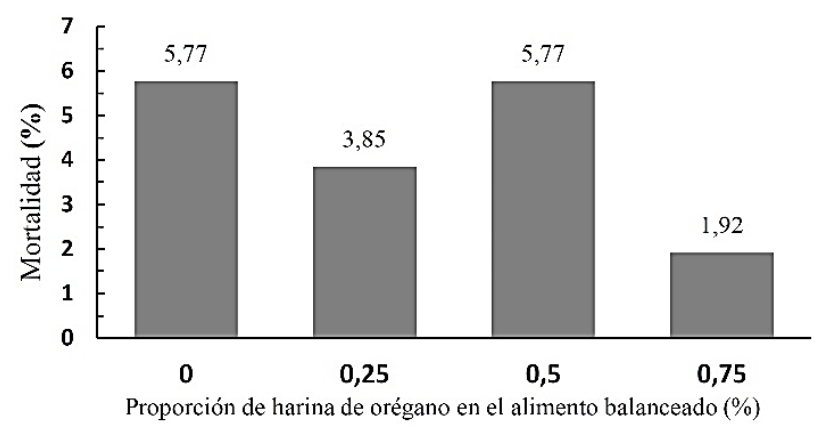

Figura 7. Histograma para el porcentaje de mortalidad en los tratamientos con la inclusión de harina de orégano en el alimento balanceado.
A diferencia del resto de las variables cuantificadas, donde existe una condición favorable asociada a la dieta con $0,25 \%$ de $\mathrm{HHO}$ y un detrimento del rendimiento en dosis superiores de este aditivo ( 0,5 y $0,75 \%$ de $\mathrm{HHO})$, la mortalidad no muestra tendencia definida y la disparidad de los valores pudiese estar vinculada a efectos climáticos de estrés calórico (Álvarez, 2012; Corona, 2012).

\section{CONCLUSIONES}

La adición entre de 0,25 y $0,26 \%$ de harina de hojas de orégano (HHO), como aditivo fitogénico en la dieta de pollos de engorde, tuvo efecto positivo sobre el peso corporal, ganancia diaria de peso, consumo de alimento y conversión alimenticia.

El tratamiento con $0,75 \%$ de $\mathrm{HHO}$ en la dieta, expresó el menor consumo diario de alimento y ganancia diaria de peso con valores de $44,70 \mathrm{~g}$ y $26,88 \mathrm{~g}$ en la fase de inicio y $103,05 \mathrm{~g}$ y $48,43 \mathrm{~g}$ en la fase de engorde, respectivamente.

\section{LITERATURA CITADA}

Álvarez J. 2012. Manejo para disminuir la mortalidad en pollos de engorde. Rev. Cub. Cie. Agri. 2(1):125132.

Arcila-Lozano, C. C., Loarca-Piña, G., Lecona-Uribe S., González de Mejía, E. 2004. El orégano: propiedades, composición y actividad biológica de sus componentes. Archivos Latinoamericanos de Nutrición 54(1):100-111.

Ávila G. 1990. Alimentación de las aves. Trillas, Ciudad de México, México, pp. 17-18.

Ayala L, Martínez L, Acosta A, Dieppa O, Hernández L. 2006. Una nota acerca del efecto del orégano como aditivo en el comportamiento productivo de pollos de ceba. Rev. Cub. Cienc. Agríc. 40(4):455-458.

Barragán J, Cepero R, Zalduendo D, Losa R. 2004. Evaluación de la eficacia zootécnica en cebo de broilers de un aditivo basado en aceites esenciales. XLI Symposium Científico de Avicultura. Barcelona, España, pp. 171-179.

Botsoglou N, Paneri P, Christaki E, Fletouri D, Spais A. 2002. Effect of dietary oregano essential oil on performance of chickens and on iron-induced lipid oxidation of breast, thigh and abdominal fat tissues. Br. Poult. Sci. 43(1):223-230.

Carro M, Ranilla M. 2002. Los aditivos antibióticos promotores del crecimiento de los animales. Situación actual y posibles alternativas. Departamento de Producción Animal. España. Disponible en línea en: http://www.produccionanimal.com.ar/informacion _t ecnica/invernada_promotores_crecimiento/01- 
$\underline{\text { aditivos antibioticos promotores.pdf }}$

(Acceso 18.02.2020).

Corona L, J. L. 2012. Impacto del estrés calórico en la producción de pollos de engorde de Venezuela. Rev. Electron. Vet. 13(6):1-9.

Campozano-Marcillo G, Hurtado E, Arteaga Ch, PérezBello A, García-Díaz J, Garzón-Jarrin R. 2020. Aceite esencial de orégano (Origanum vulgare L) y sexo como factores en la respuesta productiva en pollos de engorde. Rev. prod. anim., 33(1):

Chang S, Xian R, Myong H, Jing W, Hai J, Shu G, Bontempo V, Guang H. 2017. Effect of dietary oregano powder supplementation on the growth performance, antioxidant status and meat quality of broiler chicks. Ital. J. Anim. Sci. 16(2):246-252.

CLIMATE-DATA.ORG. 2020. Datos climáticos mundiales (en línea). Consultado 10 ene. 2021 Disponible en:https://es.climate-data.org/

Devriese L, Daube G, Hommez J, Haesebrouck F. 1993. In vitro susceptibility of Clostridium perfringens isolated from farm animals to growth enhancing antibiotics. Journal of Applied Bacteriology, 75(1): 55-57 1993.

Dewick P. 1997. Medicinal natural products. A biosyntetic approach. John Wiley \& Sons, Texas, Estados Unidos, pp. 152-213.

Díaz S. 2015. Botanical alternatives to antibiotics for use in organic poultry production. Poult. Sci. 94(2):1419-1430.

Di Rienzo J, Balzarini M, González L, Casanoves F, Tablada M, Robledo C. 2011. InfoStat Software Estadístico. InfoStat Group, College of Agricultural Sciences, National University of Córdoba: Argentina. Disponible en línea en: http://www.infostat.com.ar/ (Acceso 14.02.2020).

Eidelsburger U, and Kirchgessner M. 1994. Effects of organic acids and salts in the feed on fattening performance of broilers. Arch. Geflugelk. 58:268267.

Garcés C, Soler M, Barragán J. 2009. ¿Ejercen los extractos vegetales un efecto positivo sobre Broilers enfermos? Albéitar. España. Disponible en línea en: http://albeitar.portalveterinaria.com/noticia/8911/art iculos-nutricion-archivo/ejercen-los-extractosvegetales-un-efecto-positivo-sobre-broilersenfermos.html (Acceso 19.02.2020).

González G, Salado S, García M. 1999. Uso de aditivos como mejoradores de la calidad de las dietas para monogástricos: enzimas y acidificantes. Encuentro

\section{ERSPAMACIENCIA}

sobre Nutrición y Producción de Animales Monogástricos: Memorias. Maracay, Venezuela, pp. 6-11.

Hernández F, Madrid J, García V, Orenjo J, Megías M. 2004. Influencia de tres extractos de plantas en el rendimiento de los pollos de engorde, digestibilidad y tamaño del órgano digestivo. Poult. Sci. 83(2):169174.

Kamel C. 2001. Tracing modes of action and the roles of plant extracts in non-ruminants. Recent advances in animal nutrition. John Wiley \& Sons, Texas, Estados Unidos, pp: 135-150.

Karimi A, Yan F, Coto C, Park J, Min Y, Lu C, Gidden J, Lay J, Waldroup P. 2010. Effects of level and source of oregano leaf in starter diets for broiler chicks. J. Appl. Poult. Res. 19:137-145.

Kokkini S, Karousou R, Dardioti A, Krigas N, Lanaras T. 1997. Aceites esenciales de otoño de orégano griego. Phytochem. 44(5): 883-886.

Lara C, Márquez E, Martín R, Martín M, Navarro S. 2009. El Pollo Campero. España. Disponible en línea en: http:/www.uclm.es/profesorado/produccionanimal/ ProduccionAnimalII (Acceso 15.03.2020).

Lara P, Itzá M, Aguilar E, Sanginés J. 2010. Harinas de hojas de plantas aromáticas como fitoterapéuticos en pollos de engorda. Pesq. Agropec. Bras. 45(3):294298.

Lee K, Everts H, Kappert H, Yeom K, Beynen A. 2003. Dietary carvacrol lowers body weight gain but improves feed conversion in female broiler chickens. J. Appl. Poult. Res. 12(1):394-399.

Mchan F, Shotts E. 1992. Relationship of fatty acid production and lowered ph on the recovery of salmonella typhimurium in chicks fed selected carbohydrate. Poult. Sci. 71:80-86.

OMS (Organización Mundial de la Salud). 2017. Lista OMS de antimicrobianos de importancia crítica para la medicina humana (Lista OMS de AIC) Disponible en línea en: https://www.who.int/foodsafety/publications/cia201 7es.pdf (Acceso 11.05.2020).

Ordoñez R, E. M., Del Carpio R, P. A., Cayo C, I. S. 2018. Suplementación alimenticia con orégano (Origanum vulgare) y complejo enzimático en pollos de carne: I. Indicadores Productivos. UCV - HACER: Revista de Investigación y Cultura, 7(1): 31-44.

Ortisi F. 2008. Breve revisión sobre promotores de crecimiento. Costa Rica. Disponible en línea en: http://docplayer.es/342832-Breve-revision-sobre- 
promotores-de-crecimiento.html

(Acceso 15.04.2020).

Pinchasov Y, Jensen L. 1989. Effect of short-chain fatty acids on voluntary feed of broilers chicks. Poul. Sci. 68(12):1612-1618.

Pujada A, Vega-Vilca1 J, Velásquez V, PalaciosRodríguez B. 2019. Niveles de orégano (Origanum vulgare) en la dieta y su influencia en el rendimiento productivo del pollo de engorde. Rev Inv. Vet Perú, 30(3): 1077-1082. http://dx.doi.org/10.15381/rivep.v30i3.16599

Puvaca N, Luka D, Stanacev V, Kostadinovic L, Beukovic M, Ljuboyevic D, Zec S. 2014. Effect of spice herbs in broiler chicken nutrition on productive performances. XVI International Symposium "Feed Technology".
Quintana P. 1993. Propiedades curativas del orégano (Origanum vulgare). Ibalpe, Bogotá, Colombia, pp. 14-18.

Roldán G. 2004. Manual de Explotación de Aves de corral. Grupo Latino, Bogotá, Colombia, pp. 65.

Ringuelet J, Viña S. 2013. Productos Naturales y Vegetales. $1^{\circ}$ Edición. Facultad de Ciencias Agrarias y Forestales. Universidad Nacional de La Plata. Editorial de la Universidad de La Plata, La Plata, Argentina. 258p

Windisch, W. M., K. Schedle, C. Plitzner, and A. Kroismayr. 2008. Use of phytogenic products as feed additives for swine and poultry. J. Anim. Sci., 86: 140-148. 